\title{
Comparison of $0.5 \%$ Lignocaine with Tramadol and with Nalbuphine for Day Care IVRA in Upper Limb: an Interventional Study
}

\author{
Dr. Vishma K, Dr. Divya Vincent
}

\begin{abstract}
Background and objectives: IVRA is a good alternative to brachial block in upper limb surgeries for day-care patients posted for minor hand and forearm surgeries. We compare two adjuvants to $0.5 \%$ lignocaine in 30 patients. The objective was to determine the difference if any, in the analgesic contribution of tramadol versus nalbuphine as adjuvants.

Methods:In this randomized double-blind study, 90 patients aged 20-60 years, ASA I-II, both sexes, who were posted for minor hand and forearm surgeries in Fr. Muller Medical College Hospital were allotted into three groups of 30 each: group $N$ received lignocaine $0.5 \%$ and10 mg nalbuphine mixture, group $T$ received lignocaine $0.5 \%$ with $100 \mathrm{mg}$ tramadol and group L with plain lignocaine 0.5\%.The onset, duration of both sensory and motor blocks, time of first analgesic request postoperatively and complications were recorded.

Results:There was a statistically significant early onset of action with significant prolongation in the mean duration of sensory and motor blocks in group $T$ and group $N$ compared with group $L(P<0.001)$. The results observed in both groups were comparable. There was an increase in the mean time of first analgesic request in group T and group N compared with group L $(P<0.001)$.

Conclusion:Nalbuphine and tramadol as adjuvants to lignocaine in IVRA resulted in early onset and prolongation of the duration of both sensory and motor blocks with insignificant complications. nalbuphine had the longest duration time of postoperative analgesia.
\end{abstract}

Keywords :IVRA, Biers Block, lignocaine, tramadol-nalbuphine adjuvants

\section{Introduction}

Intravenous regional anaesthesia (IVRA) was first described by August Bier in 1908. He observed that when local anaesthetic was injected intravenously between two tourniquets on a limb, a rapid onset of anaesthesia occurred in the area between the tourniquets and a slower onset occurred beyond the distal tourniquet.

IVRA is technically straightforward and does not require specific anatomical knowledge. Published series report successful anaesthesia in $96-100 \%$ of patients with a low incidence of side-effects. It is a reliable, simple and safe method of providing anaesthesia for minor surgical procedures to the extremities if it is administered by experienced anaesthesiologist.

Local anaesthetic diffuses into the small veins surrounding the nerves and then into the vasa nervorum and capillary plexus of the nerves, leading to a core to mantle (centrifugal) conduction block in the nerves involved. Local anaesthetic then diffuses into the small nerves in the skin, blocking their conduction. The tourniquet produces ischaemia, which contributes to the analgesic action of the local anaesthetic by blocking nerve conduction and motor endplate function. 20 minutes after tourniquet application alone there will be analgesia to pinprick without the injection of any local anaesthetic.

IVRA is used for surgical interventions on the hand, forearm or elbow that will not exceed 1 hour. These include manipulation of forearm fractures, excision of wrist ganglia and palmar fasciotomy. IVRA is particularly useful for tendon grafting because it enables the surgeon to observe movement and tension of the grafted tendon (after deflating the tourniquet) before closing the wound as opposed to a regional block. IVRA can also be used for surgery on the foot, ankle or lower leg, for example for removing plates, screws or foreign bodies.

There are many adjuvants used in conjunction with IVRA for increasing the analgesic effects and providing patient comfort.

Tramadol is a weak mu receptor agonist opioid with a central analgesic effect, possesses a local anesthetic effect by blocking sodium channels at the peripheral nerve endings. It also results in the release of serotonin and inhibits the reuptake of serotonin.

Nalbuphine is a mixed k-agonist- $\mu$-antagonist opioid of the phenanthrene series. It is chemically related to naloxone and oxymorphone. It activates the spinal and supraspinal opioid receptors leading to 
adequate analgesia with cardiovascular stability,minimal sedation, less respiratory depression, less physical dependence, as well as less nausea and vomiting ${ }^{6}$

Here we compare the contribution of tramadol and nalbupine as adjuvants in IVRA in patients posted for surgeries of hand/forearm, when the surgery requires less than an hour.Additives to local anaestheticshave not been consistently shown to have an effect during IVRA but may increase the length of postoperative analgesia, probably because of a systemic effect following tourniquet release.

In this study we determine the effect of adding either tramadol or nalbuphine on the duration of motor and sensory blockade and the post operative analgesia, as compared to only local anaesthetic.

\section{Materials and methods}

The study group comprised of patients admitted to Father Muller Medical College Hospital, Mangalore, Karnataka, posted for minor hand and forearm surgeries during June-August 2016.

A prospective, interventional randomized controlled single blinded clinical study, using a sample size of 90 , with sample comprising men and women in more or less equal proportion, the sampling procedure being

Group L: 30 patients receiving IVRA with $0.5 \%$ lignocaine $40 \mathrm{ml}$

Group T: 30 patients receiving IVRA with tramadol $100 \mathrm{mg}$ and $0.5 \%$ lignocaine $40 \mathrm{ml}$

Group N: 30 patients receiving IVRA with nalbuphine $10 \mathrm{mg}$ and $0.5 \%$ lignocaine $40 \mathrm{ml}$

a) Inclusion criteria:

-Men and women between the ages of 18-60.

-ASA I-II

b) Exclusion criteria:

- Patients who are being treated with drugs that compromise the study

- Pregnant women

- Patients with neurological diseases like MS, ALS, syringomyelia, etc.

-Patients with deformities of the upper limb

- Emergency procedures.

- allergy to study drugs

After institutional ethical committee clearance from Fr Muller Medical College Hospital, Mangalore, 90 patients ,falling within the inclusion criteria will be selected for the study after Pre-anesthetic evaluation and written informed consent will be taken. Patients will be randomly allotted into the above mentioned three groups. Randomization will be done using sealed envelope technique. The two drugs will be sourced from the same company to avoid manufacturing differences.

Patients will be blinded to the study. A baseline recording of the haematological parameters - non invasive blood pressure(NIBP), Heart rate (HR) will be taken at the time of pre-anesthetic evaluation(previous day), and patients will be familiarized with the visual analogue scale(VAS) for pain assessment ( 0 being no pain and 10 being the worst possible pain). They will be asked to stay nil per oral (NPO) from 12 midnight and antacid (Ranitidine $150 \mathrm{mg}$ at night and the next morning (minimum $2 \mathrm{hrs}$ before the surgery) will be administered by the nurse in charge.

On the day of the surgery, after the patient has been shifted to the operating room, monitors will be connected for - non invasive blood pressure(NIBP), Heart rate (HR)-Electrocardiography, Oxygen saturation using standard pulse oximeter( $\mathrm{SpO} 2)$. A baseline recording will be taken again.

The baseline sensory level was assessed by a simple pinprick test with a $25-\mathrm{G}$ sterile needle to the forearm and hand on a three-point scale $(2=$ normal sensation, $1=$ blunted sensation, and $0=$ absence of sensation)]. The pain score was assessed on 10-point verbal rating scale (VRS, $0-10$, where 10 is the maximum imaginable pain, whereas 0 states complete absence of pain)

After securing an 18 or 20 gauge cannula, and ensuring free flow of fluid through the vein, patients will be preloaded with $15 \mathrm{ml} / \mathrm{kg}$ of either Ringer Lactate (RL) or Normal Saline (NS) depending on the diabetic status).

The equipment required for IVRA included:

- Pneumatic tourniquet (checked for leaks before the procedure) and a pressure gauge

- Esmarch bandage or Rhys-Davis exsanguinator

- Localanaesthetic solution

- Resuscitation equipment and drugs.

III. IVRA of the arm 
A $22 \mathrm{G}$ cannula was placed intravenously as distal as possible in the arm to be anaesthetized. Venous access was established in the opposite arm to allow administration of fluids or drugs if necessary. The double tourniquet (two tourniquets each $6 \mathrm{~cm}$ wide) or a single one (14 cm wide) was applied on the arm with generous layers of padding, ensuring that no wrinkles were formed and the tourniquet edges did not touch the skin, upper limb exanguinated with esmarch bandage.

The Esmarch bandage was then removed. Proper function of the tourniquet was assessed clinically by disappearance of the radial pulse and was confirmed by the absence of pulse-oximeter waves on the monitor. The randomly assigned patients received an intravenous injection of a local anesthetic mixture according to its selected group.

The 22-G cannula was removed after injecting the local anesthetic solution. The block was allowed to take effect. The onset of both sensory and motor blocks was recorded. The onset time for sensory block (time started from injection of local intravenous mixture until a complete sensory block was achieved in all dermatomes, sensory score $=0$ ) was assessed every $30 \mathrm{~s}$ after injection of the local anesthetic mixture using a standardized pinprick technique with a $22-\mathrm{G}$ short beveled needle. The sensation was tested in the dermatomal distribution of the lateral, medial, antebrachial cutaneous, radial, median, and ulnar nerves. The onset time for motor block was defined as the time elapsed from injection of local anesthetic mixture till achieving a complete motor block.

The motor power was tested by asking the patient to extend and flex his wrist and fingers at 30-s intervals, and complete motor block was recorded when no voluntary movement was present. After complete sensory and motor block, the distal cuff was inflated to $50 \mathrm{mmHg}$ above systolic blood pressure, $15 \mathrm{~min}$ after the inflation of the proximal one and then the proximal cuff was deflated gradually. Continuous observation of the distal tourniquet was performed to avoid unnoticed slow deflation. The minimum time for distal cuff deflation was kept not less than 30 min after inflation and was not allowed to be left inflated for more than 60 min. Pain secondary to tourniquet was assessed at a 5-min interval using VAS (where $0=$ no pain and $10=$ worst pain imaginable). If VASwas at least 4 , fentanyl $(1 \mu \mathrm{g} / \mathrm{kg})$ was given intravenously to relieve the pain. At the end of the operation, the tourniquet was deflated gradually, and the radial pulsation was checked clinically and confirmed by appearance of pulse oximeter waves. The mean blood pressure (MBP), mean heart rate, and $\mathrm{SpO}_{2}$ were recorded for all patients.

Oxygen mask was placed on the patient, delivering oxygen at $5 \mathrm{~L} / \mathrm{min}$.

Operating room staff were kept unaware of the study. Intra operative and post-operative evaluation was as follows.

NIBP , HR, SpO2 was constantly monitored throughout the surgical procedure.

Hypotension ( $>20 \%$ of baseline fall or $<90 \mathrm{mmhg}$ SBP) was treated with $6 \mathrm{mg}$ of mephenteramine with co loading of IV fluids. Dosing was repeated if required.Bradycardia( $\mathrm{HR}<50 \mathrm{bpm}$ ) was treated with IV atropine 0.3-0.6 mg, this was repeated if required. Desaturation $(\mathrm{SpO} 2<95 \%)$ was dealt by supplementing oxygen and ensuring free airway.

After the patient was shifted to the post operative ward, the NIBP, HR, SpO2, Ramsey Sedation score and VAS were recorded, every hour till recovery.

Patients complaining of nausea or who had vomiting were given injection ondansetron $0.15 \mathrm{mg} / \mathrm{kg}$ i.v .In case of complaints of pain, IV tramadol $50 \mathrm{mg}$ rescue doses was given.

\section{Statistical analysis}

Power analysis from similar studies suggest that a sample size of 30 patients/group was required to get the power of study to $80 \%$, with 0.05 level of significance. All the data was fed into the IBM SPSS software , mean and standard deviation was used for continuous data and median for non parametric data. The two groups were compared using analysis of variance (ANOVA). The sedation was compared with the help of Wilcoxon rank-sum test/ $t$ test. $\mathrm{P}$ values of $<0.05$ and Confidence intervals of $>95$ if achieved was considered significant.

Results

Table 1: Comparision of the three groups

\begin{tabular}{|c|c|c|c|c|c|c|}
\hline & \multicolumn{6}{|c|}{ GROUP } \\
\hline & \multicolumn{2}{|c|}{ CONTROL } & \multicolumn{2}{|c|}{ TRAMADOL } & \multicolumn{2}{|c|}{ NALBUPHINE } \\
\hline & Mean & $\begin{array}{l}\text { Standard } \\
\text { Deviation }\end{array}$ & Mean & $\begin{array}{l}\text { Standard } \\
\text { Deviation }\end{array}$ & Mean & $\begin{array}{l}\text { Standard } \\
\text { Deviation }\end{array}$ \\
\hline age & 39.80 & 9.90 & 36.30 & 10.68 & 38.70 & 7.93 \\
\hline $\begin{array}{l}\text { onset of sensory block in } \\
\text { seconds }\end{array}$ & 5.00 & 1.15 & 4.10 & .88 & 3.30 & .67 \\
\hline $\begin{array}{l}\text { onset of motor-complete } \\
\text { block in seconds }\end{array}$ & 9.00 & 1.33 & 7.90 & .74 & 8.50 & 1.58 \\
\hline duration of sensory block in & 74.00 & 6.99 & 84.00 & 6.99 & 84.50 & 8.32 \\
\hline
\end{tabular}


Comparison Of 0.5\% Lignocaine With Tramadol And With Nalbuphine For Day Care Ivra ...

\begin{tabular}{|l|l|l|l|l|l|l|}
\hline minutes & & & & & & \\
\hline $\begin{array}{l}\text { duration of motor block In } \\
\text { minutes }\end{array}$ & 91.50 & 8.18 & 100.00 & 8.16 & 103.00 & 8.56 \\
\hline time for rescue analgesia & 121.00 & 8.76 & 151.00 & 9.94 & 219.00 & 28.85 \\
\hline RSS & 2.20 & .63 & 2.20 & .63 & 2.30 & .48 \\
\hline
\end{tabular}

Comparison of the age distribution among the three groups, revealed that the difference was not statistically significant.Comparison of onset of sensory in seconds between the three groups shows that CONTROL group has the highest value of $5 \mathrm{~s}$ and NALBUPHINE has the least value of $3.3 \mathrm{~s}$. This difference is statistically Significant with a test value of 8.491 and $p$ value of 0.001 .

Comparing CONTROL and NALBUPHINE groups shows a mean difference of $1.700^{*}$ and is statistically significant with a $p$ value of 0.001 .

This shows that nalbuphine group had the earliest onset of sensory blockade followed by Tramadol group.

Comparison of onset of motor-complete block in seconds between the three groups shows that CONTROL group has the highest value of $9 \mathrm{~s}$ and TRAMADOL has the least value of $7.9 \mathrm{~s}$. This difference is statistically Insignificant with a test value of 1.887 and $p$ value of 0.171 . This shows that the difference in onset of complete motor blockade is not significant among the three groups.

Comparison of duration of sensory block in minutes between the three groups shows that NALBUPHINE group has the highest value of $84.5 \mathrm{~min}$ and CONTROL has the least value of $74 \mathrm{~min}$. This difference is statistically Significant with a test value of 6.304 and $p$ value of 0.006 .

This shows that nalbuphine provided the longest duration of sensory blockade, followed by tramadol.

Comparison of duration of motor block in min between the three groups shows that NALBUPHINE group has the highest value of $103 \mathrm{~min}$ and CONTROL has the least value of $91.5 \mathrm{~min}$. This difference is statistically Significant with a test value of 5.158 and $p$ value of 0.013

This shows that nalbuphine group provided longest duration of motor blockade, followed by tramadol.

Comparison of time for rescue analgesia between the three groups shows that NALBUPHINE group has the highest value of $219 \mathrm{~min}$ and CONTROL has the least value of $121 \mathrm{~min}$. This difference is statistically Significant with a test value of 16.421 and $\mathrm{p}$ value of $<0.001$.

This shows that nalbuphine provided longest duration of analgesia followed by tramadol.

Comparison of Ramsay sedation score ${ }^{11}$ (RSS) between the three groups shows that NALBUPHINE group has the highest value of 2.3 and CONTROL has the least value of 2.2. This difference is statistically Insignificant with a test value of 0.097 and $\mathrm{p}$ value of 0.908 .

This shows that there was good sedation among nalbuphine and tramadol groups.
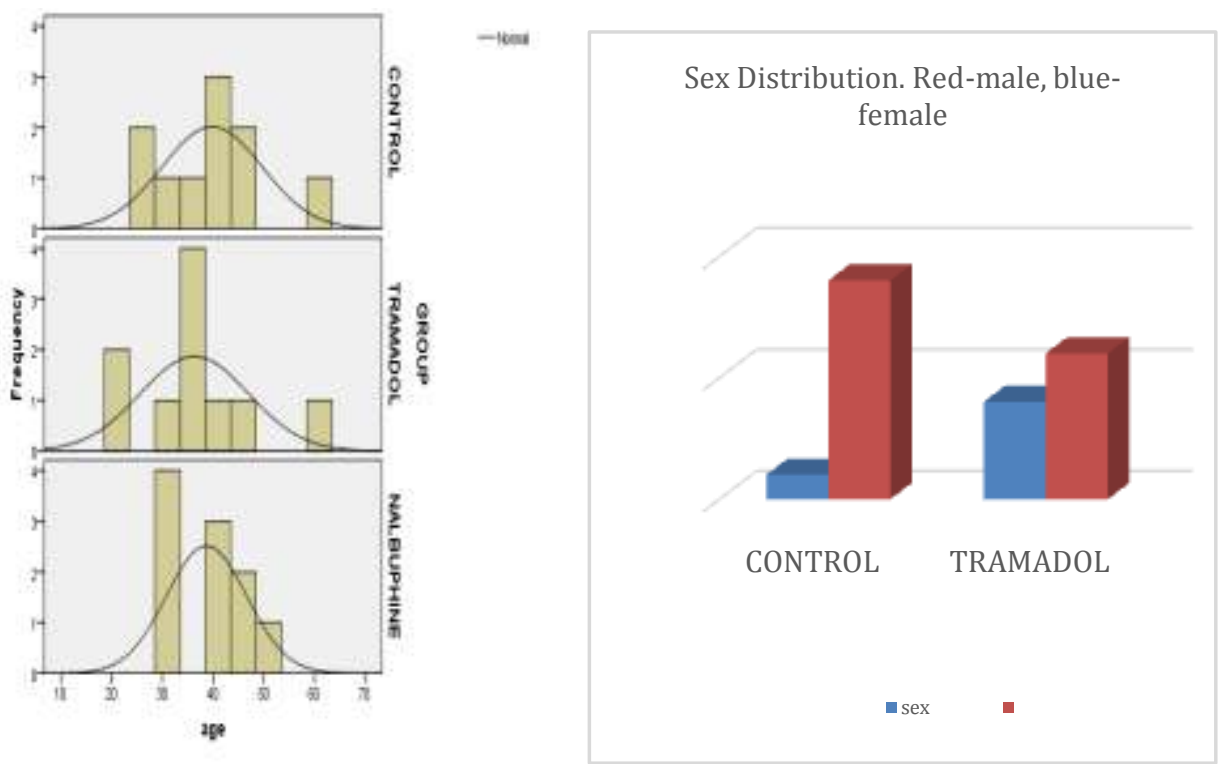

Graph 1: Histogram representing age distribution among the three groupsGraph 2: Distribution of sex 


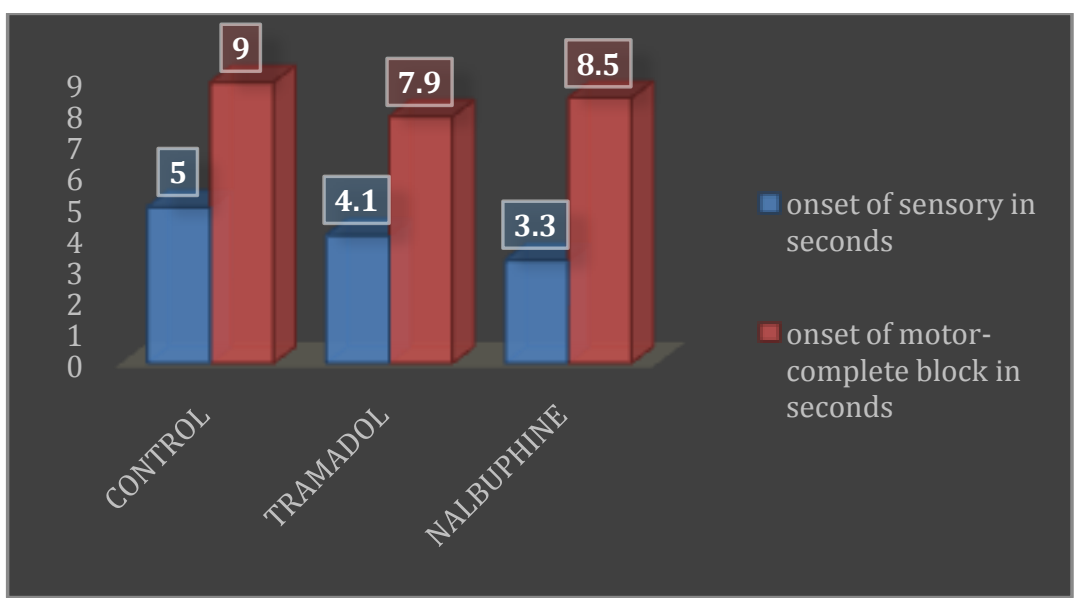

Graph 3: Onset of sensory blockade in the three groups

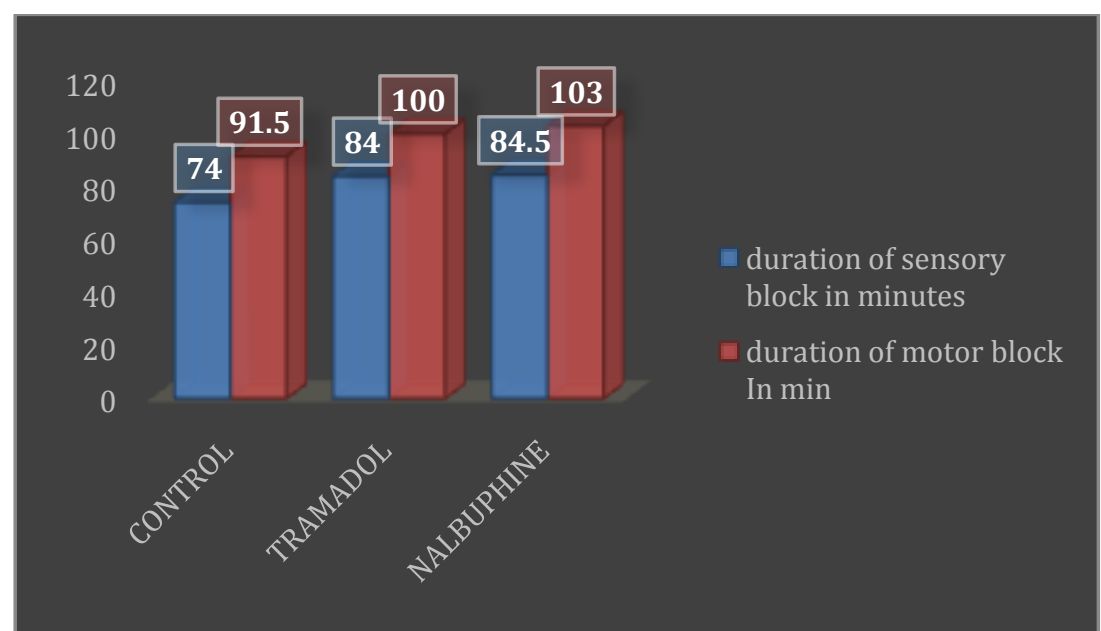

Graph 4: Duration of motor and sensory blockade among the three groups

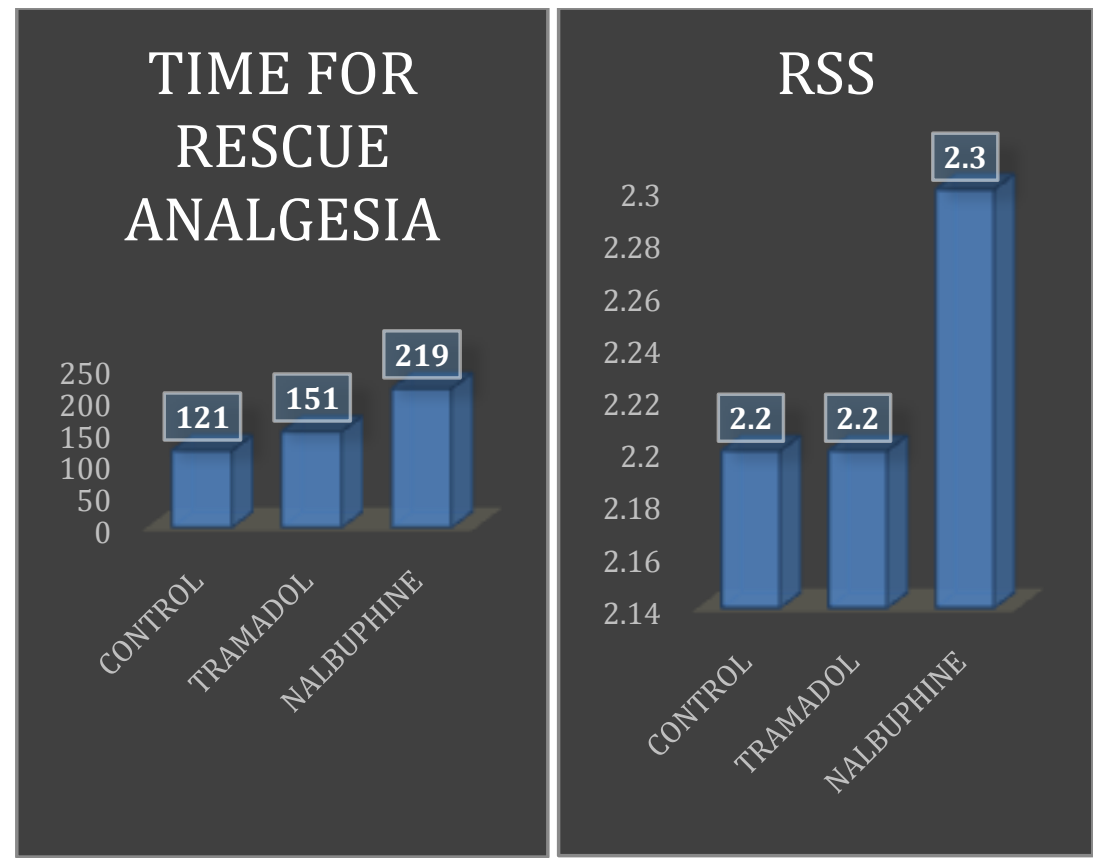

Graph 5: Time for rescue analgesiaGraph 6: Ramsay Sedation Score among the three groups 
Table 2: Comparison of the three groups

\begin{tabular}{|c|c|c|c|c|c|}
\hline Dependent Variable & (I) group & (J) group & $\begin{array}{l}\text { Mean } \\
\text { Difference } \\
(1-J)\end{array}$ & $\begin{array}{l}\text { Std. } \\
\text { Error }\end{array}$ & $\begin{array}{l}P \\
\text { VALUE }\end{array}$ \\
\hline \multirow[t]{3}{*}{ age } & \multirow[t]{2}{*}{ CONTROL } & TRAMADOL & 3.5 & 4.281 & 0.696 \\
\hline & & NALBUPHINE & 1.1 & 4.281 & 0.964 \\
\hline & TRAMADOL & NALBUPHINE & -2.4 & 4.281 & 0.842 \\
\hline \multirow{3}{*}{$\begin{array}{l}\text { onset of sensory in } \\
\text { seconds }\end{array}$} & \multirow[t]{2}{*}{ CONTROL } & TRAMADOL & 0.9 & 0.413 & 0.093 \\
\hline & & NALBUPHINE & $1.700^{*}$ & 0.413 & do01 \\
\hline & TRAMADOL & NALBUPHINE & 0.8 & 0.413 & 0.147 \\
\hline \multirow{3}{*}{$\begin{array}{l}\text { onset of motor- } \\
\text { complete block in } \\
\text { seconds }\end{array}$} & \multirow[t]{2}{*}{ CONTROL } & TRAMADOL & 1.1 & 0.567 & 0.147 \\
\hline & & NALBUPHINE & 0.5 & 0.567 & 0.656 \\
\hline & TRAMADOL & NALBUPHINE & -0.6 & 0.567 & 0.548 \\
\hline \multirow{3}{*}{$\begin{array}{l}\text { duration of sensory } \\
\text { block in minutes }\end{array}$} & \multirow[t]{2}{*}{ CONTROL } & TRAMADOL & $-10.000^{*}$ & 3.336 & $\underline{\underline{0.015}}$ \\
\hline & & NALBUPHINE & $-10.500^{*}$ & 3.336 & $\underline{0.011}$ \\
\hline & TRAMADOL & NALBUPHINE & -0.5 & 3.336 & 0.988 \\
\hline \multirow{3}{*}{$\begin{array}{l}\text { duration of motor } \\
\text { block In min }\end{array}$} & \multirow[t]{2}{*}{ CONTROL } & TRAMADOL & -8.5 & 3.714 & 0.075 \\
\hline & & NALBUPHINE & $-11.500^{*}$ & 3.714 & $\underline{0.012}$ \\
\hline & TRAMADOL & NALBUPHINE & -3 & 3.714 & $\overline{0.702}$ \\
\hline \multirow{3}{*}{$\begin{array}{l}\text { time for rescue } \\
\text { analgesia }\end{array}$} & \multirow[t]{2}{*}{ CONTROL } & TRAMADOL & $-30.000^{*}$ & 8.197 & $\underline{\underline{0.003}}$ \\
\hline & & NALBUPHINE & $-98.000^{*}$ & 8.197 & $\leq 0.001$ \\
\hline & TRAMADOL & NALBUPHINE & $-68.000^{*}$ & 8.197 & $\underline{\underline{00001}}$ \\
\hline \multirow[t]{3}{*}{ RSS } & \multirow[t]{2}{*}{ CONTROL } & TRAMADOL & 0 & 0.262 & 1 \\
\hline & & NALBUPHINE & -0.1 & 0.262 & 0.923 \\
\hline & TRAMADOL & NALBUPHINE & -0.1 & 0.262 & 0.923 \\
\hline
\end{tabular}

\section{Discussion}

In this study we enrolled 90 patients, and randomly allotted 30 in each group. Group C-30 patients.Group T- 30 patients. Group N-30 patients after informed consent. The goal of our study was to compare the effects of adjuvants like tramadol and nalbuphine on intravenous regional anesthesia, especially its effect on post-operative analgesia.For post-operative analgesia, commonly used analgesics are those that cause least respiratory depression, drowsiness, without compromising on pain relief.Tramadol was chosen because it is one of the most commonly used post operative analgesic. The idea behind adding it as an adjuvant versus intravenous form would be a more targeted distribution and analgesic effect. By adding it in the mixture of the local anesthetic, we hoped to prolong the duration of analgesia and perhaps even cause an early onset of sensory blockade, ${ }^{7}$ thereby providing better anaesthesia to the patients as well as avoid the need for a rescue analgesic, providing a smoother transition from anesthetized state to normalcy.

Nalbuphinewas chosen to be compared with tramadol because although tramadol has mu receptor agonism, it is weak compared to nalbuphine and has a theoretically superior opioid effect.It is also not as frequently used, and its potency is under-rated.Nalbuphine is a pain mediation primarily, as it is an agonist at kappa and mu receptors, having fewer behavioral effects than other opioids ${ }^{8}$

The equi-analgesic dose of tramadol $100 \mathrm{mg}$ is $10 \mathrm{mg}$ of nalbuphine ${ }^{9}$. The patients were given IVRA based on their group in doses mentioned above.They were all taught the Visual analogue scale in the pre-anesthetic check up ${ }^{10}$ They were all demographically comparable as mentioned above in the results. The onset of sensory blockade was highest in nalbuphine group, starting within 3.3 seconds, followed by tramadol group in which mean onset was 4.1 seconds and control was 5 seconds.

The onset of motor blockade was comparable in all three groups and was not statistically significant with a mean of 8-9 seconds in all the groups.

The duration of sensory blockade was highest in the nalbuphine group, 84 minutes \pm 8 minutes, compared to tramadol group which had a mean of $84 \pm 6$ minutes, followed by 74 minutes \pm 8 minutes.

The duration of motor blockade was longest in nalbuphine group, which was 103 minutes followed by tramadol group which had an average of 100 minutes and control group which had an average of 91 minutes.

Time to request for analgesic was longest in nalbuphinegroup which was 219 minutes followed by tramadol group which was 151 minutes and control group which had an average of 121 minutes. This was statistically very significant which shows that nalbuphine is superior when it comes to duration of post-operative analgesia. 
The degree of sedation was comparable among tramadol and nalbuphinegroups, which was an average of 2.3, which is more than light sedation but were easily arousable.

The hemodynamics were not compared among the three groups .

There were no significant adverse events.

\section{Conclusion}

Nalbuphine and tramadol as adjuvants to lignocaine in IVRA resulted in early onset and prolongation of the duration of both sensory and motor blocks with insignificant complications. Both tramadol and nalbuphine provided longer analgesia when compared to the control group. Nalbuphine had the longest duration of postoperative analgesia which was statistically very significant.

\section{References}

[1]. Miller RD,ErikssonLI,Fleiisher LA, Wiener-Kronish,et al. Miller's Anesthesia. Vol 1, ch 89, 2626-27.

[2]. Yektas A, GumusF ,Karayel A, AlagolA.Effects of Addition of Systemic Tramadol or Adjunct Tramadol to Lidocaine Used for Intravenous Regional Anesthesia in Patients Undergoing Hand Surgery. Anesthesiol Res Pract. 2016;2016:9161264. doi: $10.1155 / 2016 / 9161264$.

[3]. Siddiqui AK et al,Tramadol as an adjuvant to intravenous regional anesthesia with lignocaine, Saudi Med J. 2008 Aug;29(8):1151-5

[4]. BakriM .Nalbuphine as an adjuvant to IVRA .2016 ClinicalTrials.gov identifier: NCT02678585

[5]. RN Solanki et alComparative Study of Intravenous NalbuphineHCl and Tramadol HCl for Post- Operative Pain Relief Following Orthopaedic Surgeries ,Asian Pac. J. Health Sci., 2015; 2(1): 155-160

[6]. Youssef MM, ElZayyat NS. Lidocaine-nalbuphine versus lidocaine-tramadol for intravenous regional anesthesia. Ain-Shams J Anaesthesiol 2014;7:198-204

[7]. Dayer P, Desmeules J, Collart L. Pharmacology of tramadol. Journ of drugs. 1997; 53 Suppl 2:18-24

[8]. Schmidt WK,TamSW,ShotzbergerGS,Smith DH Jr, et al. Nalbuphine. Drug Alcohol Depend. 1985 Feb;14(3-4):339-62

[9]. Natusch D. Equianalgesic doses of opioids-their use in clinical practice. Br J Pain. 2012 Feb; 6(1):43-46.

[10]. Hawker GA, Mian S, Kendzerska T, French M. Measures of Adult Pain. Arthritis care and research. Vol 63 Nov 2011: 240-252.

[11]. Marino PL, Sutin KM. The ICU Book. Sedation in ICU. Table 49.3 